\title{
Scanner abdominal : étude comparative de l'exposition patient en routine clinique sur des appareils avec et sans reconstruction itérative
}

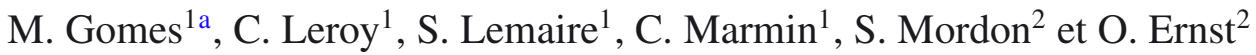 \\ 1 Service de radiologie digestive, Hôpital Claude Huriez, CHUR Lille, 1 rue Polonovski, 59037 Lille Cedex, France. \\ 2 INSERM, U703, Univ. Lille Nord de France, CHRU, 52 rue du Docteur Yersin, 59120 Loos, France.
}

Reçu le 22 décembre 2012 - Accepté le 3 juin 2013

\begin{abstract}
Résumé - Objectif : comparer la dose délivrée aux patients et la qualité d'image en routine clinique lors de la réalisation d'un scanner abdominal ne disposant pas des techniques de reconstructions itératives (RI) par rapport à un examen réalisé sur un scanner disposant des RI. Matériels et méthodes : il s'agit d'une étude rétrospective incluant 30 patients ayant eu deux examens abdominaux sur un scanner 40 coupes (TDM40) et sur un scanner 256 coupes avec RI (TDM256). Les patients, suivis pour une pathologie abdominale chronique, ont eu un examen de même indication sur chaque scanner avec un protocole comprenant une phase abdomino-pelvienne au temps portal. La longueur d'acquisition, la dose efficace et le Produit Dose Longueur (PDL) ainsi que des évaluations quantitatives et qualitatives de l'image ont été comparés. Résultats : la dose efficace moyenne pour un examen était de $17,3 \mathrm{mSv}$ avec le TDM40 (PDL : 1019 mGy.cm) contre 11,1 mSv avec le TDM256 (PDL : $654 \mathrm{mGy} . \mathrm{cm}$ ) soit une réduction de 35,8\% ( $p<0,001)$. Les longueurs d'acquisitions et l'évaluation quantitative étaient comparables dans les deux groupes. L'évaluation qualitative était légèrement supérieure sur le TDM40 mais aucun examen n'a été considéré comme sous-optimal. Conclusion : l'utilisation d'un scanner équipé de RI permet une réduction significative de la dose efficace tout en préservant une bonne qualité d'image.
\end{abstract}

\begin{abstract}
Abdominal CT scan: A comparative study of patient exposure in clinical routine on devices with and without iterative reconstruction. Objective: compare the dose delivered to patients and image quality in clinical routine to perform an abdominal CT scan with no iterative reconstruction techniques (IR) relative to an examination conducted on a scanner with IR. Materials and methods: this is a retrospective study of 30 patients who underwent two abdominal examinations: one on a 40-slice scanner (TDM40) without IR and another one on a 256-slice scanner with IR (TDM256). The patients, on medical follow-up for a chronic abdominal disease, had an exam on each scanner using the same protocol comprising an abdominopelvic time portal phase. The length of acquisition, the effective dose and the dose length product (DLP) as well as quantitative and qualitative assessments of the image were compared. Results: the average effective dose per examination was $17.3 \mathrm{mSv}$ with the TDM40 (PDL: $1019 \mathrm{mGy} . \mathrm{cm}$ ) against $11.1 \mathrm{mSv}$ with the TDM256 (PDL: 654 mGy.cm), hence a reduction of $35.8 \%(p<0.001)$. The length of acquisition and quantification were comparable in both groups. The qualitative assessment was slightly higher on the TDM40 but no examination was considered suboptimal. Conclusion: using a scanner equipped with IR significantly reduces the effective dose while maintaining image quality.
\end{abstract}

Keywords: patient dose / as low as reasonably achievable (ALARA) / computed tomography / low doses / radiation dose

\section{Introduction}

Les progrès technologiques récents ont nettement étendu les applications cliniques de la tomodensitométrie (TDM). Alors que les avantages de la TDM ont été bien documentés, l'augmentation des doses de rayonnement délivrées à la population a soulevé l'attention sur la nécessité d'une réduction

\footnotetext{
a gomes.mat@gmail.com
}

de l'exposition aux radiations lors des examens TDM (Etard et al., 2010) ce qui devient une préoccupation de santé publique. Plusieurs publications récentes ont rappelé les effets potentiels des radiations en tomodensitométrie (Einstein et al., 2007 ; Sodickson et al., 2009).

Face à cette augmentation de l'irradiation, les nouvelles technologies permettent une réduction de doses. Citons pour les améliorations à l'acquisition : la collimation dynamique 
qui réduit l'exposition en début et fin d'hélice, l'amélioration du filtrage physique, les logiciels d'adaptation du milliampérage... (Rengo et al., 2011). Pour la reconstruction des images, la principale avancée correspond aux reconstructions itératives. Cette technique consiste à appliquer des algorithmes itératifs de réduction du bruit au cours de la reconstruction des images à la fois sur les données brutes, c'est-à-dire sur les projections et sur les données de l'image (Rampado et al., 2012). Au cours de ces dernières années, un effort pour réduire la dose (principe ALARA : As-Low-As-reasonably-Achievable) en travaillant avec les algorithmes itératifs (Martinsen et al., 2012) pour la reconstruction de l'image a été engagé par la communauté médicale de radiologie.

Comme celui de toutes les autres procédures TDM, le volume annuel d'imagerie abdominale a enregistré une croissance continue, toutes les indications des examens scanographiques ne pouvant être remplacées par des examens IRM. On constate aussi une augmentation significative du nombre des examens scanographiques (+26\% entre 2002 et 2007) (Etard et al., 2010), ainsi qu'une augmentation du nombre d'acquisitions par examen (Guite et al., 2011) en particulier pour l'étude $\mathrm{du}$ foie, où les séquences quadri-phasiques sont de plus en plus fréquentes. Par ailleurs, la pathologie abdominale est particulièrement concernée par la dosimétrie, du fait de la radiosensibilité élevée des organes abdominaux et de la nécessité d'obtenir une bonne discrimination en densité. L'imagerie abdominale est donc un secteur critique, mais la qualité de l'information délivrée par les scanners multi coupes améliore nos capacités diagnostiques et le service rendu aux malades.

Les actes de diagnostic médical en France conduisent à une dose efficace de $1,3 \mathrm{mSv}$ en moyenne par an et par individu (Etard et al., 2010) en se situant au milieu de la fourchette des valeurs moyennes européennes $(0,4 \mathrm{mSv}$ au RoyaumeUni ; $2 \mathrm{mSv}$ en Belgique). Cette valeur est très inférieure à celle des États-Unis ( $3 \mathrm{mSv}$ ). Les examens scanographiques délivrent $58 \%$ de cette dose efficace moyenne. Cette augmentation concerne en particulier les examens couvrant le thorax, l'abdomen et le pelvis, c'est-à-dire des organes radiosensibles qui contribuent fortement à la dose efficace (Christner et al., 2010). Cette hausse de l'exposition médicale (Valentin, 2007) est liée à une augmentation des indications, une facilité et une rapidité des acquisitions multi coupes, une répétition des acquisitions et une disparition des acquisitions incrémentales.

L'objectif de cette étude est donc de mesurer la réduction de la dose délivrée aux patients en routine clinique lors de la réalisation d'un examen abdominal réalisé sur un scanner multi coupe installé en 2004 ne disposant pas des techniques de reconstructions itératives (RI) par rapport à un examen réalisé sur un scanner dernière génération installé en 2011 disposant des reconstructions itératives tout en comparant la qualité d'image afin de s'assurer qu'elle n'est pas altérée.

\section{Matériel et méthodes}

\subsection{Population de patients}

Il s'agit d'une étude mono centrique rétrospective incluant 30 patients ayant bénéficié de deux examens scanographiques abdominaux sur deux machines différentes. Les examens sur un scanner 40 coupes Philips Brilliance 40 (TDM40) ont été effectués entre mai 2010 et septembre 2011 alors que les examens sur le scanner dernière génération 256 coupes Philips iCT 256 Brilliance (TDM256) ont été effectués entre juillet 2011 et septembre 2011. Les images du scanner précédent étaient alors disponibles sur le PACS du service pour la comparaison.

Les patients inclus ont eu un examen abdominal de même indication sur chaque scanner. Il s'agissait d'adultes suivis pour une pathologie abdominale nécessitant des contrôles réguliers : suivis de carcinomes hépatocellulaires, de cholangiocarcinomes, de néoplasies endocriniennes multiples, de métastases hépatiques, de mélanomes, de greffes hépatiques, de tumeurs neuroendocrines, de cancers colorectaux, thyroïdiens et de cortico-surrénalome.

Pour chaque patient, l'âge et l'indice de masse corporelle (IMC) étaient notés pour chacun des deux scanners.

Le critère d'exclusion était un changement marqué du poids corporel dans l'intervalle des deux imageries (supérieur à $5 \mathrm{~kg}$ ).

\subsection{Techniques scanographiques}

Ces patients avaient bénéficié de deux examens avec un protocole similaire dont au moins une phase abdominopelvienne au temps portal.

Afin de comparer le bruit et la qualité subjective globale de l'image, nous avons analysé uniquement les images sur la phase portale.

Cent à cent cinquante millilitres d'un produit de contraste iodé concentré à $350 \mathrm{mg} / \mathrm{ml}$ étaient injectés par voie intraveineuse à l'aide d'un injecteur automatique, la phase veineuse était définie par une acquisition faite à 90 secondes (s) après l'injection de produit de contraste.

Pour chaque examen, le produit dose longueur (PDL) de la phase veineuse a été enregistré ainsi que les longueurs d'acquisition, les kilovolts $(\mathrm{kV})$ et les milliampères seconde (mAs). Ces paramètres étaient choisis par le radiologue vacataire.

Les deux scanners présentaient une modulation angulaire automatique des milliampères $(\mathrm{mA})$ par rapport à l'absorption des patients (D-DOM) (Söderberg et Gunnarsson, 2010). La moyenne de l'intensité du courant utilisée sur le TDM256 était de $163 \mathrm{mAs}$ (minimale : $100 \mathrm{mAs}$, maximale : $350 \mathrm{mAs}$ ), alors qu'elle se situait à 225 mAs sur le TDM40 (minimale : $120 \mathrm{mAs}$, maximale : $400 \mathrm{mAs}$ ).

Le temps de rotation était de $0,5 \mathrm{~s}$ pour tous les examens. Les champs de vue étaient adaptés à chaque patient sur les deux machines. L'épaisseur de reconstruction des coupes était de $1,5 \mathrm{~mm}$, mais la relecture s'est faite en coupe plus épaisse de $3 \mathrm{~mm}$.

Sur le TDM40, une tension de $140 \mathrm{kV}$ a été choisie chez 22 patients, et de $120 \mathrm{kV}$ chez 8 patients alors que tous les protocoles sur le TDM256 étaient réalisés à $120 \mathrm{kV}$.

Le TDM256 est équipé d'un logiciel de reconstructions itératives (RI) : iDose ${ }^{4}$ (Philips), où l'importance du processus de réduction de bruit dans le domaine de l'image est choisie sur une échelle de 1 à 7 . Plus le niveau est élevé plus la réduction du bruit est importante (Noël et al., 2011), dans notre étude le niveau 3 a été utilisé. 


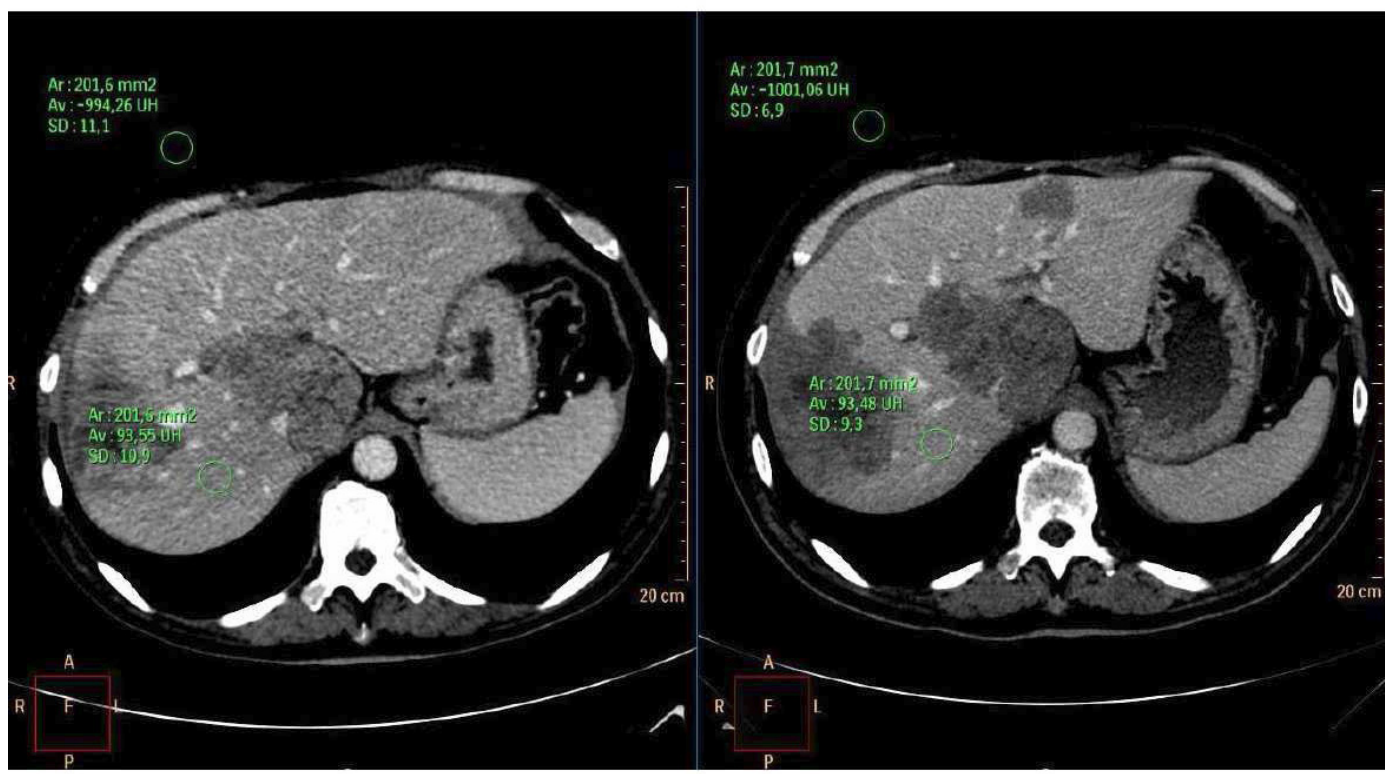

Fig. 1. Analyses d'images en étude qualitative, à gauche le TDM256, à droite le TDM40 chez un patient de 62 ans $\left(\mathrm{BMI}: 26 \mathrm{~kg} / \mathrm{m}^{2}\right)$ suivi dans le cadre de métastases hépatiques. À gauche : TDM256 à $120 \mathrm{kV}, 250 \mathrm{mAs}$ : DLP : 549,6 mGy/cm; à droite : TDM40 à $140 \mathrm{kV}, 250 \mathrm{mAs}$ : DLP : $993 \mathrm{mGy} / \mathrm{cm}$. Le bruit d'image a été défini comme l'écart-type (SD air) des valeurs des pixels d'une ROI circulaire disposée dans l'air en avant de l'hypochondre droit sur une coupe de $3 \mathrm{~mm}$ en fenêtre abdominale. Le rapport S/B du foie correspondait à la valeur d'atténuation de la ROI placée dans le parenchyme hépatique sur le bruit (densité du foie/SD air).

Analyses of image quality, the TDM256 on the left-hand side, the TDM40 on the right-hand side, for a 62 -year-old patient $\left(\mathrm{BMI} 26 \mathrm{~kg} / \mathrm{m}^{2}\right)$ followed for liver metastases. On the left: TDM 256-120 kV, $250 \mathrm{mAs}$ : DLP: $549.6 \mathrm{mGy} / \mathrm{cm}$; on the right: TDM 40 to $140 \mathrm{kV}, 250 \mathrm{mAs}$ : DLP: $993 \mathrm{mGy} / \mathrm{cm}$.

\subsection{Analyse d'images}

\section{Étude quantitative}

Les densités et le bruit ont été mesurés en unité Hounsfield (UH : unité définissant, en tomodensitométrie, l'absorption du faisceau de rayons $\mathrm{X}$ par les divers tissus qu'il traverse) dans deux régions d'intérêt (ROI) placées dans le foie et l'air pour comparer les valeurs de rapport signal sur bruit $(\mathrm{S} / \mathrm{B})$ des deux examens. Ces ROI, de $2 \mathrm{~cm}^{2}\left( \pm 10 \mathrm{~mm}^{2}\right)$, ont été placées par le même radiologue dans une région homogène et identique sur les deux examens sur une coupe axiale au dessus ou à hauteur $\mathrm{du}$ tronc porte en regard des segments IV, VII ou VIII, de $3 \mathrm{~mm}$ d'épaisseur en fenêtre abdominale (Fig. 1).

Le bruit d'image a été défini comme l'écart-type (SD air) des valeurs des pixels d'une ROI circulaire disposée dans l'air en avant de l'hypochondre droit (Fig. 1) selon la méthode décrite par Hara et al. (2009).

Le rapport $\mathrm{S} / \mathrm{B}$ du foie correspondait à la valeur d'atténuation de la ROI placée dans le parenchyme hépatique sur le bruit (densité du foie/SD air), en évitant les vaisseaux et les lésions focales.

\section{Étude qualitative}

Une évaluation qualitative de la qualité image a été effectuée grâce à un serveur d'application travail disponible dans le service (PHILIPS PORTAL) par deux radiologues digestifs d'expérience en imagerie hépatobiliaire.
Cette évaluation de qualité d'image globale (Figs. 2, 3) a été réalisée sur les coupes axiales natives de $3 \mathrm{~mm}$ en fenêtre hépatique sur une échelle allant de 0 à $5(0=$ examen ininterprétable; 1 = image de qualité médiocre $; 2=$ image de qualité moyenne; 3 = image de bonne qualité ; 4 = image de très bonne qualité ; 5 = image d'excellente qualité). Les notes 0,1 et 2 étaient considérées comme une image pouvant altérer la capacité diagnostique.

Cette évaluation a été réalisée indépendamment par chaque radiologue, en aveugle, après anonymisation, suppression de l'affichage des paramètres d'acquisition et avec une répartition des examens au hasard.

\subsection{Mesure de la longueur d'acquisition}

La longueur d'acquisition était exprimée en cm et correspondait à la différence de position entre la première et la dernière image de l'acquisition au temps veineux.

\section{5 Évaluation de la dose d'irradiation}

La dose délivrée était directement fournie par le rapport d'examen. Elle correspondait au PDL exprimé en mGy.cm.

La dose efficace $\mathrm{E}(\mathrm{mSv})$ a été calculée par la formule $: \mathrm{E}=\mathrm{Epdl} \times \mathrm{PDL}$ (Bongartz et al., 1999), où Epdl $\left(0,017 \mathrm{mSv} \cdot \mathrm{mGy}^{-1} \cdot \mathrm{cm}^{-1}\right)$ correspondait au coefficient de conversion du PDL en dose efficace moyennée pour l'abdomen et le pelvis (Bongartz et al., 1999). 


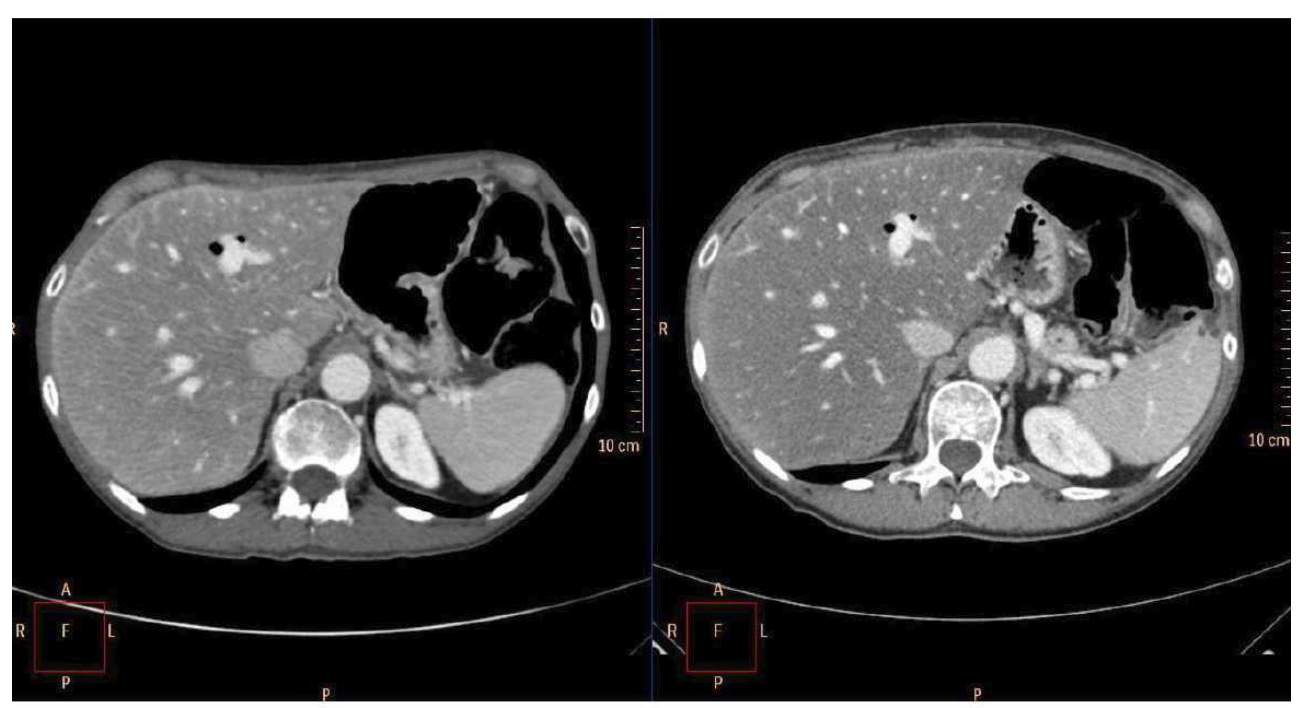

Fig. 2. Patiente de 69 ans $\left(B M I: 17,4 \mathrm{~kg} / \mathrm{m}^{2}\right)$ suivi après une duodéno-pancréatectomie céphalique pour adénocarcinome du pancréas; à gauche : TDM256, $120 \mathrm{kV}, 150 \mathrm{mAs}$ : DLP : $360 \mathrm{mGy} / \mathrm{cm}$; à droite : TDM40, $140 \mathrm{kV}, 120 \mathrm{mAs}$ : DLP : 588,1 mGy/cm. À gauche, le bruit de l'image parait discrètement augmenté dans le foie, cependant la qualité d'image est toujours maintenue pour la caractérisation des lésions. Case of a 69-year-old patient (BMI : $17.4 \mathrm{~kg} / \mathrm{m}^{2}$ ) followed after cephalic duodeno-pancreatectomy for adenocarcinoma of the pancreas. On the left: TDM256, $120 \mathrm{kV}, 150 \mathrm{mAs}$ : DLP $360 \mathrm{mGy} / \mathrm{cm}$; on the right: TDM40, $140 \mathrm{kV}, 120 \mathrm{mAs}$ : DLP: $588.1 \mathrm{mGy} / \mathrm{cm}$.

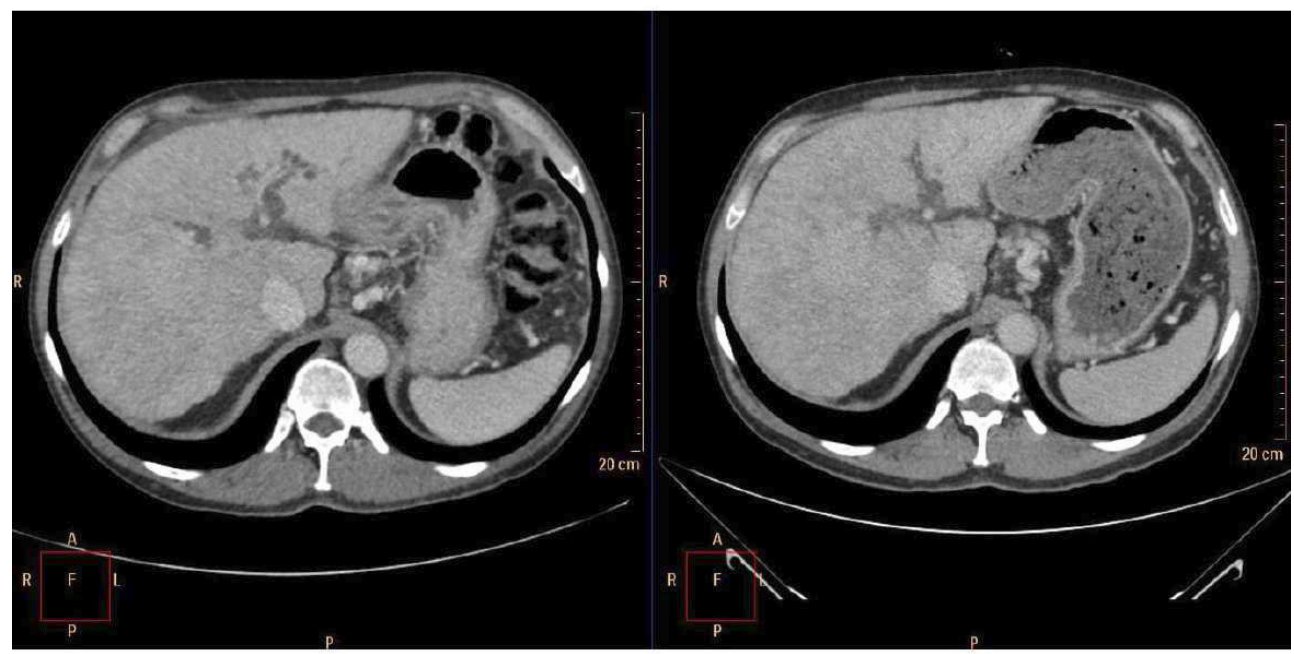

Fig. 3. Patient de 57 ans (BMI 25,3 kg/m²) suivi dans le cadre d'un carcinome hépatocellulaire. À gauche : TDM256, 120 kV, 150 mAs, DLP : $528 \mathrm{mGy} / \mathrm{cm}$; à droite : TDM40, $140 \mathrm{kV}, 220 \mathrm{mAs}$ : DLP : $976 \mathrm{mGy} / \mathrm{cm}$. Notez la qualité d'image comparable entre les images malgré la réduction de $46 \%$ de la dose.

Case of a 57 -year-old patient (BMI $25.3 \mathrm{~kg} / \mathrm{m}^{2}$ ) followed for a hepatocellular carcinoma. On the left: TDM256, $120 \mathrm{kV}, 150 \mathrm{mAs}$, DLP: 528 $\mathrm{mGy} / \mathrm{cm}$; on the right: CT40, $140 \mathrm{kV}, 220 \mathrm{mAs}$ : DLP: $976 \mathrm{mGy} / \mathrm{cm}$.

\subsection{Analyse statistique}

Un test de Student apparié a été utilisé pour comparer les valeurs de PDL, de dose efficace, de longueur d'acquisition, de bruit, de rapport $\mathrm{S} / \mathrm{B}$ et de qualité d'image subjective entre les deux groupes.

Les données ont été analysées en utilisant le logiciel «primer of biostatistics » version 4.02 .

Une valeur $p$ de moins de 0,05 a été considérée comme statistiquement significative.

\section{Résultats}

La moyenne d'âge était de 57 ans (de 23 à 78 ans) et l'IMC moyen de $25,4 \mathrm{~kg} / \mathrm{m}^{2}$ (de 17,4 à $34,5 \mathrm{~kg} / \mathrm{m}^{2}$ ). La population était représentée par 8 femmes et 22 hommes. La moyenne de l'intervalle entre les deux imageries pour le même patient était de 5 mois ( 1 mois à 16 mois).

Les différentes valeurs quantitatives sont résumées dans le tableau 1.

Il n'y avait pas de différence significative entre la longueur d'acquisition (Tab. 2). Le rapport signal sur bruit n'est pas significativement différent entre les 2 machines (Tab. 1). À 
Tableau 1. Évaluation de la qualité de l'image entre les deux scanners.

Evaluation of image quality between two scanners.

\begin{tabular}{lccc}
\hline & TDM40 & TDM256 & Valeurs de $\boldsymbol{p}$ \\
\hline Mesures quantitatives & & & \\
\hline $\begin{array}{l}\text { Ecart-type de la ROI } \\
\text { dans le foie (UH) }\end{array}$ & 12,48 & 12,71 & $p=0,928$ \\
\hline Estimation du bruit (UH) & 31,6 & 25,7 & $p=0,208$ \\
\hline Rapport signal sur bruit & 8,7 & 11,1 & $p=0,147$ \\
\hline Qualité de l'image & & & \\
\hline Radiologue 1 & 3,93 & 3,90 & $p=0,57$ \\
\hline Radiologue 2 & 4,26 & 3,96 & $p=0,037$ \\
\hline
\end{tabular}

Tableau 2. Évaluation de la dose délivrée entre les deux scanners et des longueurs d'acquisitions.

Evaluation of the radiation dose issued between two scanners and length of acquisition.

\begin{tabular}{lccc}
\hline & Scanner 40 & Scanner 256 & Valeurs de $\boldsymbol{p}$ \\
\hline PDL (mGy.cm) & 1019,2 & 654,1 & $p<0,001$ \\
\hline Dose efficace $(\mathrm{mSv})$ & 17,3 & 11,1 & $p<0,001$ \\
\hline $\begin{array}{l}\text { Longueur } \\
\text { d'acquisition }(\mathrm{cm})\end{array}$ & 46,5 & 45,8 & $p=0,163$ \\
\hline
\end{tabular}

l'inverse, le PDL et la dose efficace étaient de 35,8\% inférieurs de façon significative (Tab. 2) sur l'appareil avec les reconstructions itératives (TDM256).

Pour l'analyse qualitative (Tab. 1), un des radiologues ne retrouvait pas de différence significative entre les deux scanners alors que le second radiologue trouvait une différence significative en faveur du groupe TDM40.

Cependant, pour les deux lecteurs, quel que soit l'appareil, aucun examen n'a été coté en dessous de la note 3 (image de bonne qualité), donc aucune imagerie n'a été considérée comme sous-optimale pour l'interprétation diagnostique.

\section{Discussion}

Notre étude confirme que l'utilisation d'un scanner récent avec RI permet de réduire de manière significative la dose de rayonnement tout en conservant une qualité d'image diagnostique (Noël et al., 2011).

Plusieurs paramètres peuvent expliquer l'amélioration de la dosimétrie avec le TDM256.

- Premièrement une surface d'acquisition du détecteur plus étendue qui permet de limiter la surgénération de faisceaux en augmentant l'efficacité des doses sur l'axe Z. Cette réduction s'explique par une moindre importance de l'effet d'overbeaming.

- Deuxièmement, l'utilisation d'un collimateur dynamique qui s'ouvre automatiquement au début des acquisitions hélicoïdales et se ferme à la fin, permettant de limiter l'exposition du patient au dessus et en dessous de la région explorée (Van der Molen et Geleinjs, 2007).

- Ensuite, l'amélioration du filtrage du faisceau X avec l'utilisation de filtres papillons qui facilite l'administration uniforme de la dose et la répartition égale du bruit au cours de la rotation du tube en fonction de la morphologie du patient.

- L'amélioration du système de modulation automatique du courant (Kalra et al., 2004) en fonction du morphotype du patient.

- Et enfin, l'utilisation d'un algorithme de RI (iDose ${ }^{4}$ ) (Noël et al., 2011) sophistiqué et complexe. L'algorithme iDose ${ }^{4}$ comprend un traitement itératif du signal au niveau des données brutes associé à un traitement d'image complexe pour diminuer le bruit en préservant la résolution spatiale à contraste élevé.

Le traitement approprié du bruit, tel que prévu par iDose 4 à un niveau de signal très faible peut réduire le bruit de projection et par conséquent les artéfacts.

Il commence dans le domaine des projections où il identifie et corrige les mesures tomodensitométriques les plus bruitées, c'est-à-dire celles qui ont un mauvais rapport $\mathrm{S} / \mathrm{B}$ ou un nombre de photons trop faible. Par un processus itératif (Qiu et al., 2010), les données bruitées sont pénalisées, et les bords sont préservés. Le bruit qui reste après cette étape est propagé à l'espace image.

L'étape suivante est la soustraction du bruit dans le domaine de l'image tout en préservant les bords sous-jacents associés à l'anatomie réelle. Une sélection est faite parmi le modèle qui correspond le mieux à la topographie locale pour réduire le bruit du volume de l'image choisie.

Dans la littérature, les doses délivrées sur le TDM256 et le TDM40 sont comparables à celles d'autres études sur des appareils sans et avec RI, en effet Nakaura et al. (2011) avaient une moyenne de PDL de 567,8 ( $\pm 152 \mathrm{mGy} . \mathrm{cm})$ sur la phase veineuse du foie à $120 \mathrm{kV}$ avec RI sur un TDM 256 coupes.

Alors que la majorité des examens était effectuée à $140 \mathrm{kV}$ sur le TDM40, tous les examens étaient réalisés à $120 \mathrm{kV}$ sur le TDM256, ce qui est concordant avec plusieurs études qui conseillent la diminution des $\mathrm{kV}$ lors de l'emploi des RI. Marin et al. (2010) qui trouvaient une dose efficace de $17,5 \mathrm{mSv}$ en RPF avec une tension de 140 kV à l'aide d'un TDM 64 coupes, ont conclu que la RI peut améliorer la qualité de l'image avec l'utilisation d'une haute tension plus faible et un courant plus élevé sur un examen abdominal.

Notre diminution de dose de $35,8 \%$ est également proche des études comparant des examens sans et avec RI en tomodensitométrie abdominale (Prakash et al., 2010). Sagara et al. (2010) montraient une réduction du PDL de $33 \%$ sur le même scanner 64 coupes avec RI et retrouvaient une dose de rayonnement à $1193 \mathrm{mGy}$ soit $18 \mathrm{mSv}$ en rétroprojection filtrée (RPF). Ces chiffres sont comparables à notre étude, et également proches de ceux de Schindera et al. (2011) qui ont montré une réduction de dose de $39 \%$ en RI à une tension de $100 \mathrm{kV}$ par rapport à la RPF à $120 \mathrm{kV}$ sans augmentation du bruit.

De plus, cette réduction de dose nous permet une moyenne de PDL bien inférieure (654,1 mGy.cm) au nouveau niveau de référence diagnostique 2012 en scanographie pour une acquisition abdomino-pelvienne ( $800 \mathrm{mGy} . \mathrm{cm})$ chez l'adulte (JORF, 2012).

Concernant les données qualitatives, Sagara et al. (2010) retrouvaient une image plus nette en RPF et une image plus bruitée en RI en particulier chez les patients en surpoids $($ BMI > 25) de façon significative, mais avec une capacité 
diagnostique comparable à la RI, comme d'autres auteurs (May et al., 2011).

Dans notre étude, un des lecteurs avait aussi une différence significative en faveur de la RPF. Cependant cette différence de qualité restait faible, et aucun examen ne donnait une qualité sousoptimale comme dans l'étude de Sagara et al. (2010).

Cela ne correspond pas à une baisse du rapport S/B. Il s'agit donc probablement d'un rendu différent de l'image (Figs. 2, 3). Il pourrait peut-être s'agir d'une augmentation du flou, ce paramètre n'ayant pas été étudié. Le rendu différent peut être aussi lié à la différence de tension utilisée sur les deux machines, en particulier dû aux artéfacts de durcissement du faisceau à proximité du rachis qui peut aussi expliquer l'aspect différent malgré la reconstruction itérative en raison du plus bas kV utilisé sur le TDM256. Ainsi, pour le TDM40, une tension de $140 \mathrm{kV}$ a été utilisée chez 22 patients.

En dépit de la diminution de la dose, le rapport S/B n'est pas diminué (Heyer et al., 2007). Sagara et al. (2010) retrouvaient une mesure du bruit dans le parenchyme hépatique diminuée en RI de manière significative, alors que dans notre étude la réduction du bruit n'était pas significative en RI.

Notre étude comporte certaines limites.

Tout d'abord, cette série est rétrospective et comporte un faible effectif de patients inclus ce qui peut expliquer que le rapport $\mathrm{S} / \mathrm{B}$ et la réduction du bruit ne soient pas significatifs.

En second lieu, nos critères qualitatifs d'évaluation de l'image sont également discutables. Nous avons opté pour la mesure du bruit de l'image à partir de la déviation standard d'une ROI disposée dans l'air autour du patient, comme cela a déjà été décrit par plusieurs auteurs (Hara et al., 2009; Sagara et al., 2010). Toutefois, le bruit de l'image n'est pas un critère suffisant et d'autres critères, plus difficilement analysables, participent également à appréhender la qualité d'image comme par exemple les artéfacts, le contraste ou la résolution spatiale pouvant expliquer la différence de qualité retrouvée chez un des lecteurs.

Le TDM40 imposait d'employer $140 \mathrm{kV}$ chez la majorité des patients pour conserver un rapport signal sur bruit suffisant, alors qu'une tension de $120 \mathrm{kV}$ était suffisante sur le TDM256. Cette différence peut modifier l'aspect des images entre les deux techniques.

À noter que la réduction de la tension de 140 à $120 \mathrm{kV}$ permet d'améliorer le contraste par une augmentation de la densité de l'iode et donc de réduire la dose à qualité d'image équivalente.

L'évaluation qualitative d'une image n'est pas non plus une méthode très facile à mettre en œuvre. Il aurait fallu différentier plusieurs critères sur les données qualitatives, la résolution spatiale, le bruit, le rapport $\mathrm{S} / \mathrm{B}$, les artéfacts, la notion d'interprétabilité.

Ce qui est intéressant dans notre étude, c'est la comparaison entre deux systèmes de reconstructions avec deux scanners de générations différentes sur les mêmes patients, ce qui n'a pas été encore été réalisé à notre connaissance. Il y a eu une volonté claire de ne pas modifier la qualité image. Malgré une absence de dégradation de la qualité d'image, on observe une importante réduction de la dose d'irradiation en routine clinique. Il est probablement possible de diminuer immédiatement la dosimétrie en acceptant un niveau de bruit plus élevé, ce qui n'a pas encore été fait sur le site pour ce type d'examen.

\section{Conclusion}

En conclusion, l'utilisation en pathologie abdominale d'un scanner nouvelle génération, équipé de 256 détecteurs et de reconstructions itératives, permet une réduction de la dose efficace de $35,8 \%$, avec un niveau de bruit et un rapport signal sur bruit similaire à celui d'un scanner de génération antérieure sans RI sur les mêmes patients dans les mêmes conditions cliniques.

La qualité subjective est légèrement inférieure probablement du fait d'un rendu d'image différent, le rapport signal sur bruit n'étant pas diminué.

Dans l'avenir, une amélioration des techniques de reconstruction et l'acceptabilité d'une baisse modérée du rapport $\mathrm{S} / \mathrm{B}$ devraient encore permettre de diminuer nettement la dosimétrie de chaque examen, sans diminution de l'efficacité diagnostique.

\section{Références}

Bongartz G. et al. (1999) Guidelines on Quality Criteria for Computed Tomography. Brussels, Belgium : European Commission, Report EUR 16262 EN, 70.

Christner J.A., Kofler J.M., McCollough C.H. (2010) Estimating effective dose for CT using dose-length product compared with using organ doses : consequences of adopting ICRP Publication 103 or dual-energy scanning, Am. J. Roentgenol. 194 (4), 881889.

Einstein A.J., Henzlova M.J., Rajagopalan S. (2007) Estimating risk of cancer associated with radiation exposure from 64-slice computed tomography coronary angiography, J. Am. Med. Assoc. 298 (3), 317-323.

Etard C., Sinno-Tellier S., Aubert B. (2010) L'exposition de la population française aux rayonnements ionisants liée aux actes de diagnostic médical en 2007, Institut de veille sanitaire (InVS) et l'Institut de radioprotection et de sûreté nucléaire (IRSN), pp. 17$18,34-37$.

Guite K.M. et al. (2011) Ionizing radiation in abdominal CT : unindicated multiphase scans are an important source of medically unnecessary exposure, J. Am. Coll. Radiol. 8 (11), 756-761.

Hara A.K. et al. (2009) Iterative reconstruction technique for reducing body radiation dose at CT : feasibility study, Am. J. Roentgenol. 193 (3), 764-771.

Heyer C.M., Mohr P.S., Lemburg S.P., Peters S.A. (2007) Image quality and radiation exposure at pulmonary CT angiography with $100-$ or $120-\mathrm{kVp}$ protocol : prospective randomized study, Radiology 245 (2), 577-583.

JORF (2012) Arrêté du 24 octobre 2011 relatif aux niveaux de référence diagnostiques en radiologie et en médecine nucléaire. Journal officiel de la république française du 14 janvier 2012.

Kalra M.K. et al. (2004) Comparison of Z-axis automatic tube current modulation technique with fixed tube current $\mathrm{CT}$ scanning of abdomen and pelvis, Radiology 232 (2), 347-353.

Marin D. et al. (2010) Low-tube-voltage, high-tube-current multidetector abdominal CT : improved image quality and decreased radiation dose with adaptive statistical iterative reconstruction algorithm-initial clinical experience, Radiology 254 (1), 145-153. 
Martinsen A.C.T., Sæther H.K., Hol P.K., Olsen D.R., Skaane P. (2012) Iterative reconstruction reduces abdominal CT dose, Eur. J. Radiol. 81 (7), 1483-1487.

May M.S. et al. (2011) Dose reduction in abdominal computed tomography : intraindividual comparison of image quality of full-dose standard and half-dose iterative reconstructions with dual-source computed tomography, Invest. Radiol. 46 (7), 465-470.

Nakaura T. et al. (2011) Abdominal dynamic CT in patients with renal dysfunction : contrast agent dose reduction with low tube voltage and high tube current-time product settings at 256-detector row CT, Radiology 261 (2), 467-476.

Noël P.B. et al. (2011) Initial performance characterization of a clinical noise-suppressing reconstruction algorithm for MDCT, Am. J. Roentgenol. 197 (6), 1404-1409.

Prakash P. et al. (2010) Reducing abdominal CT radiation dose with adaptive statistical iterative reconstruction technique, Invest. Radiol. 45 (4), 202-210.

Qiu W. et al. (2010) New iterative cone beam CT reconstruction software : parameter optimisation and convergence study, Comput. Methods Programs Biomed. 100 (2), 166-174.

Rampado O., Bossi L., Garabello D. (2012) Characterization of a computed tomography iterative reconstruction algorithm by image quality evaluations with an anthropomorphic phantom, Eur. J. Radiol. 81 (11), 3172-3177.
Rengo M. et al. (2011) The optimal contrast media policy in CT of the liver. Part I : Technical notes, Acta Radiol. 52 (5), 467-472.

Sagara Y. et al. (2010) Abdominal CT : comparison of low-dose CT with adaptive statistical iterative reconstruction and routinedose CT with filtered back projection in 53 patients, Am. J. Roentgenol. 195 (3), 713-719.

Schindera S.T. et al. (2011) Iterative reconstruction algorithm for abdominal multidetector $\mathrm{CT}$ at different tube voltages : assessment of diagnostic accuracy, image quality, and radiation dose in a phantom study, Radiology 260 (2), 454-462.

Söderberg M., Gunnarsson M. (2010) Automatic exposure control in computed tomography-an evaluation of systems from different manufacturers, Acta Radiol. 51 (6), 625-634.

Sodickson A. et al. (2009) Recurrent CT, cumulative radiation exposure, and associated radiation-induced cancer risks from CT of adults, Radiology 251 (1), 175-184.

Valentin J. (2007) Managing patient dose in multi-detector computed tomography (MDCT), ICRP Publication 102.

Van der Molen A.J., Geleijns J. (2007) Overranging in multisection $\mathrm{CT}$ : quantification and relative contribution to dose-comparison of four 16-section CT scanners, Radiology 242 (1), 208-216.

Cite this article as: M. Gomes, C. Leroy, S. Lemaire, C. Marmin, S. Mordon, O. Ernst. Scanner abdominal : étude comparative de l'exposition patient en routine clinique sur des appareils avec et sans reconstruction itérative. Radioprotection 49(1), 35-41 (2014). 